TRANSACTIONS OF THE

AMERICAN MATHEMATICAL SOCIETY

Volume 360, Number 7, July 2008, Pages 3473-3492

S 0002-9947(08)04554-6

Article electronically published on February 27, 2008

\title{
GENERALIZED RECIPROCITY LAWS
}

\author{
JOSÉ M. MUÑOZ PORRAS AND FERNANDO PABLOS ROMO
}

\begin{abstract}
The aim of this paper is to give an abstract formulation of the classical reciprocity laws for function fields that could be generalized to the case of arbitrary (non-commutative) reductive groups as a first step to finding explicit non-commutative reciprocity laws. The main tool in this paper is the theory of determinant bundles over adelic Sato Grassmannians and the existence of a Krichever map for rank $n$ vector bundles.
\end{abstract}

\section{INTRODUCTION}

In this paper we offer a unified approach to several geometric reciprocity laws, such as the Weil reciprocity law for the tame symbol and reciprocity laws for the Contou-Carrère symbol, in a way susceptible to being generalized to the non-abelian case.

The main tool used in our formulation of reciprocity laws is the algebraic theory of infinite determinants (as in [5]). The analytic counterpart of the theory has been developed by A. Pressley, G. Segal and G. Wilson ([12, 13]), and we follow the algebraic theory developed in [1] and 2]. An important difference with the paper of A. Beilinson, S. Bloch and H. Esnault ([5]) is the fact that we do not introduce a super-structure on the determinant bundles.

The determinant bundles over the Sato Grassmannian induce a central extension of the restricted linear group $\mathrm{Gl}(\infty)$. These central extensions induce central extensions of the loop groups, whose commutators allow us to recover the "classical symbols" up to a sign $(\S 2)$.

When $X$ is a complete, smooth and connected curve over a perfect field $k$ with function field $\Sigma_{X}$, adele ring $\mathbb{A}_{X}$, and $G \subset \operatorname{Gl}(n, k)$ a reductive algebraic group over $k$, we obtain a family of central extensions:

$$
\begin{gathered}
1 \rightarrow \mathbb{G}_{m} \rightarrow \widetilde{G}_{x} \longrightarrow G_{x} \rightarrow 1, \\
1 \rightarrow k^{*} \rightarrow \widetilde{G}_{\mathbb{A}_{X}} \longrightarrow G_{\mathbb{A}_{X}} \rightarrow 1, \\
1 \rightarrow k^{*} \rightarrow \widetilde{G_{\Sigma_{X}}} \longrightarrow G_{\Sigma_{X}} \rightarrow 1,
\end{gathered}
$$

where $G_{x}$ is the algebraic loop group over $k(x)$ (for each closed point $x \in X$ ); $G_{\mathbb{A}_{X}}$ is the adelic group, and $G_{\Sigma_{X}}$ is the group defined over $\Sigma_{X}$. Our main result (Theorem 3.5) can be stated by saying that the extension (1.3) is trivial. In the case $G=\mathbb{G}_{m}$, this statement is equivalent to the Weil reciprocity law, as is proved

Received by the editors March 8, 2006.

2000 Mathematics Subject Classification. Primary 14H05, 19F15, 14M15.

This work was partially supported by DGI research contract no. MTM2006-07618 and Castilla y León regional government contract SA071/04.

(C)2008 American Mathematical Society Reverts to public domain 28 years from publication 
in Remark 3.7. The proof of the main result is an easy consequence of the existence of a Krichever map for rank $n$ vector bundles over $X$ with formal trivialization at closed points.

Let $\{,\}_{A_{x}},\{,\}_{\mathbb{A}_{X}}$ and $\{,\}_{\Sigma_{X}}$ be the pairings defined by the commutators of the extensions (1.1), (1.2) and (1.3), respectively. The pairing $\{f, g\}_{\mathbb{A}_{X}}$ (for two commuting elements $f, g \in G_{\mathbb{A}_{X}}$ ) is not exactly the product $\prod_{x \in X}\{f, g\}_{A_{x}}$. The precise relation (stated in $\S 3$ for $G=\mathbb{G}_{m}$, and in $\S 4$ for a non-commutative $G)$ is that $\{f, g\}_{\mathbb{A}_{X}}=(\operatorname{sign}) \cdot \prod_{x \in X}\{f, g\}_{A_{x}}$. This allows the recovery of the classical tame symbol in terms of the commutator without imposing an extra super-bundle structure on the determinant bundles.

The last section of the paper is devoted to exploring the reciprocity laws obtained in the non-commutative case $G=\mathrm{Gl}(2, k)$. We make explicit computations in some examples and prove that the corresponding reciprocity laws are a consequence of the abelian one. Of course, it remains to study more general examples of commuting elements of $\mathrm{Gl}\left(2, \Sigma_{X}\right)$, which could give new identities.

For contrast, here we do not impose the triviality of the central extension (1.3) (Theorem 3.5) in terms of the cocycles associated with some section of the map $\widetilde{G}_{\mathbb{A}_{X}} \longrightarrow G_{\mathbb{A}_{X}}$.

We hope that further research in these directions could open a way to find explicit non-abelian reciprocity laws. To finish this Introduction, it is important to mention the very suggestive paper of E. Witten ([16]), in which the multiplicative Ward identities of a Quantum Field Theory are interpreted in terms of the triviality of the central extension (1.3).

\section{Preliminaries}

2.A. Functor of points. Firstly, we shall recall the concept of $S$-valued points in the theory of schemes: if $Y$ and $S$ are $k$-schemes, an $S$-valued point of $Y$ is a morphism of schemes from $S$ to $Y$. The set of all such points is denoted

$$
Y^{\bullet}(S)=\operatorname{Hom}_{k-s c h .}(S, X) .
$$

The functor

$$
Y^{\bullet}: \mathcal{C}_{k-s c h .} \rightarrow \mathcal{C}_{\text {sets }}
$$

is called the "functor of points" of the $k$-scheme $Y$, and the set $Y^{\bullet}(\operatorname{Spec} k)$ consists of the "rational points of $Y$ ". We should remark that in this sense each $k$-scheme can be considered as a functor from the category of $k$-schemes to the category of sets (or to the category of abelian groups when $Y$ is a group scheme).

In this work we shall write $Y(S)$ instead of $Y^{\bullet}(S)$.

2.B. Determinant bundles and central extensions of $\mathrm{Gl}(\infty)$. Let $V$ be a $k$ vector space endowed with a structure of a linear topological vector space and let us assume that $V$ is complete with respect to this topology. We shall also assume that a basis, $\tau$, of the topology of $V$ is given by the family of vector subspaces $A$ of $V$, which are commensurable (in the Tate sense [2], 4, 14]) with a fixed vector subspace $V_{+} \subset V$. We use the symbol $A \sim V_{+}$to denote commensurable vector subspaces. 
In this paper we will be mainly interested in two examples:

- $V=k((z))$ and $V_{+}=k[[z]]$.

- $V=\mathbb{A}_{X}$, the ring of adeles of a smooth curve $X$ over $k$.

Given the pair $\left(V, V_{+}\right)$we can construct a Grassmannian, $\operatorname{Gr}(V)$, which is a $k$-scheme ([2], Th. 2.13). This scheme is the algebraic version of the Sato Grassmannian. The rational points of $\operatorname{Gr}(V)$ are the discrete vector subspaces of $V$ : that is, the vector subspaces $U \subseteq V$ such that $U \cap V_{+}$and $V / U+V_{+}$are finitedimensional vector spaces over $k$. The index of a rational point $U \in \operatorname{Gr}(V)(\operatorname{Spec} k)$ is:

$$
i_{U}=\operatorname{dim}_{k}\left(U \cap V_{+}\right)-\operatorname{dim}_{k}\left(V / U+V_{+}\right),
$$

and the concept of index can be extended to arbitrary points of $\operatorname{Gr}(V)$.

Denoting by $\operatorname{Gr}_{d}(V)$ the subscheme over which the index takes values equal to $d \in \mathbb{Z}$, one can show that the $\operatorname{Gr}_{d}(V)$ are open connected subschemes, and the decomposition of $\operatorname{Gr}(V)$ in connected components is:

$$
\operatorname{Gr}(V)=\coprod_{d \in \mathbb{Z}} \operatorname{Gr}_{d}(V)
$$

Given a vector subspace $A \subset V$ commensurable with $V_{+}$(i.e. such that $\left.\operatorname{dim}_{k}\left(A+V_{+} / A \cap V_{+}\right)<\infty\right)$, there exists a complex of modules over $\operatorname{Gr}(V)$ :

$$
\mathcal{C}_{A}^{\bullet} \equiv 0 \rightarrow \mathrm{L} \oplus \widehat{A}_{\mathrm{Gr}(V)} \stackrel{\delta}{\longrightarrow} \widehat{V}_{\mathrm{Gr}(V)} \rightarrow 0,
$$

$\mathrm{E}$ being the universal subspace of $\widehat{V}_{\mathrm{Gr}(V)}$ and $\delta$ being the addition map (see 2], section 3 for details). $\mathcal{C}_{A}^{\bullet}$ is a perfect complex and its determinant in the sense of Knudsen-Mumford ([9]) is denoted by $\operatorname{Det}_{A}$ and is a line bundle over $\operatorname{Gr}(V)$. Given a rational point $U \in \operatorname{Gr}(V)(k)$, the fibre of the bundle $\operatorname{Det}_{A}$ over $U$ is:

$$
\operatorname{Det}_{A}(U) \simeq \Lambda(U \cap A) \otimes \Lambda(V / U+A)^{*},
$$

$\Lambda$ being the maximal exterior power.

The bundle $\operatorname{Det}_{V_{+}}$will be denoted simply by Det.

Remark 2.1. Kapranov has defined the notion of a determinantal theory over a locally linearly compact $k$-vector space (8, Definition 1.2.2). The determinantal theories on $V$ and their isomorphisms form a category (groupoid) denoted by $\operatorname{Det}(V)$. In our setting, the groupoid $\operatorname{Det}(V)$ can be identified with the set of line bundles:

$$
\left\{\operatorname{Det}_{A}, A \sim V_{+}\right\} .
$$

Note that the line bundles $\operatorname{Det}_{A}$ are all isomorphic ([2], Theorem 3.5).

The restricted linear group, $\mathrm{Gl}(V)$, of $\left(V, V_{+}\right)$is the contravariant functor over the category of $k$-schemes defined by:

$$
S \rightsquigarrow \operatorname{Gl}(V)(S)=\left\{g \in \operatorname{Aut}_{\emptyset_{S}}\left(\widehat{V}_{S}\right) \text { such that } g \text { is bicontinuous }\right\}
$$

(see 2], section 4, for precise definitions). This group acts naturally on $\operatorname{Gr}(V)$. In the case $V=k((z))$, the set of rational points of the restricted linear group is precisely the Japanese group $\mathrm{Gl}(\infty)$.

Let us denote by $\mathrm{Gl}_{0}(V)$ the subfunctor of $\mathrm{Gl}(V)$ that preserves the connected components of $\operatorname{Gr}(V)$. One can easily prove that $g^{*}$ Det $\simeq$ Det for every $g \in \mathrm{Gl}_{0}(V)$. 
Let us define $\widetilde{\mathrm{Gl}_{0}}(V)$ as the set of commutative diagrams:

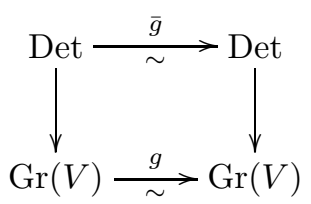

for every $g \in \mathrm{Gl}_{0}(V)$. $\widetilde{\mathrm{Gl}_{0}}(V)$ is a functor of groups and there is a natural homomorphism $\pi: \widetilde{\mathrm{Gl}_{0}}(V) \rightarrow \mathrm{Gl}_{0}(V)$ defined by $\pi(\bar{g})=g$.

In ([2], section 3) it is proved that $H^{0}\left(\operatorname{Gr}_{0}(V), \varnothing_{\mathrm{Gr}_{0}(V)}\right)=k$. This implies that there exists a canonical central extension of the functor of groups:

$$
1 \rightarrow \mathbb{G}_{m} \rightarrow \widetilde{\mathrm{Gl}_{0}}(V) \rightarrow \mathrm{Gl}_{0}(V) \rightarrow 1 .
$$

From the definitions, one has a natural action of $\widetilde{\mathrm{Gl}_{0}}(V)$ on $\left.\operatorname{Det}\right|_{\operatorname{Gr}_{0}(V)}$ and also on Det.

Let us now consider the case $V=k((z))$ and $V_{+}=k[[z]]$, and let $\sigma$ denote the product by $z^{-1}$ in $V$ (the "shift operator"). $\sigma$ induces an automorphism of $\operatorname{Gr}(V)$, also denoted by $\sigma$, and obviously $\sigma$ is a rational point of $\operatorname{Gl}(V)$. For each $d \in \mathbb{Z}, \sigma$ is an isomorphism $\sigma: \operatorname{Gr}_{d}(V) \stackrel{\sim}{\longrightarrow} \operatorname{Gr}_{d-1}(V)$. Let us denote by $\widetilde{\sigma}$ a lifting of $\sigma$ to Det:

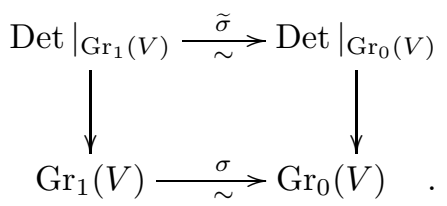

Clearly, there exists such a $\widetilde{\sigma}$, and it is unique up to multiplication by elements of $\mathbb{G}_{m}$.

The isomorphism $\widetilde{\sigma}$ induces an automorphism, $\bar{\sigma}$, of the group $\widetilde{\mathrm{Gl}_{0}}(V)$ by the following formula:

$$
\bar{\sigma}(\widetilde{A})=\widetilde{\sigma} \cdot \widetilde{A} \cdot \widetilde{\sigma}^{-1}
$$

The automorphism $\sigma$ generates a group subscheme, $\langle\sigma\rangle$, of $\mathrm{Gl}(V)$ isomorphic to $\mathbb{Z}_{*}:=\coprod_{\alpha \in \mathbb{Z}}$ Spec $k$, and there exists an isomorphism of group functors:

$$
\mathrm{Gl}(V) \simeq \mathrm{Gl}_{0}(V) \ltimes<\sigma>.
$$

The automorphism $\bar{\sigma}$ allows us to define a semidirect product:

$$
\widetilde{\mathrm{Gl}}(V): \widetilde{\mathrm{Gl}_{0}}(V) \ltimes \mathbb{Z}_{*}
$$

such that there exists an exact sequence of group functors:

$$
1 \rightarrow \mathbb{G}_{m} \rightarrow \widetilde{\mathrm{Gl}}(V) \rightarrow \mathrm{Gl}(V) \rightarrow 1
$$

From the construction it is obvious that the central extension $\widetilde{\mathrm{Gl}}(V)$ does not depend on the lifting $\widetilde{\sigma}$ of $\sigma$. For each subgroup $G \subseteq \operatorname{Gl}(V)$ the above sequence induces an exact sequence:

$$
1 \rightarrow \mathbb{G}_{m} \rightarrow \widetilde{G} \rightarrow G \rightarrow 1
$$

Let $\Gamma$ be the formal commutative group scheme representing the contravariant functor on the category of $k$-schemes:

$$
S \rightsquigarrow k((z))^{*}(S):=H^{0}\left(S, \varnothing_{S}\right)((z))^{*}
$$


(2], section 4). It is clear that $\Gamma \subset \operatorname{Gl}(V)$, and hence one has a central extension of group schemes:

$$
1 \rightarrow \mathbb{G}_{m} \rightarrow \widetilde{\Gamma} \rightarrow \Gamma \rightarrow 1 .
$$

Since $\Gamma$ is commutative, the commutator of $\widetilde{\Gamma}$ induces a pairing:

$$
\begin{aligned}
\{, \quad\}_{V_{+}}^{V}: \Gamma \times \Gamma & \longrightarrow \mathbb{G}_{m} \\
(a, b) & \longmapsto\{a, b\}_{V_{+}}^{V}=\widetilde{a} \cdot \widetilde{b} \cdot \widetilde{a}^{-1} \cdot \widetilde{b}^{-1},
\end{aligned}
$$

$\widetilde{a}$ and $\widetilde{b}$ being liftings of $a, b$ to $\widetilde{\Gamma}$. The explicit computation of the pairing $\{\quad, \quad\}_{V_{+}}^{V}$ can be found in [10].

Remark 2.2. Let $X$ be a smooth and connected curve over a perfect field $k$ and let $p \in X$ be a closed point on it. Let us consider $\Sigma_{X}$ (the function field of $X$ ), $A_{p}=\hat{\emptyset}_{p}$ (the completion of the local ring $\left.\varnothing_{X, p}\right)$ and $K_{p}=\left(\hat{\emptyset}_{p}\right)_{0}$ (the field of fractions of $\hat{\emptyset}_{p}$, which coincides with the completion of $\Sigma_{X}$ with respect to the valuation ring $\left.\varnothing_{X, p}\right)$. Since $k$ is a perfect field, one has that $A_{p} \simeq k(p)[[t]]$ and $K_{p} \simeq k(p)((t))$, $k(p)$ being the residue field of $p$. We set $\operatorname{deg}(p)=\operatorname{dim}_{k} k(p)$.

Let us consider the $k$-vector spaces $V_{+}=A_{p}$ and $V=K_{p}$, which define the Grassmannian $\operatorname{Gr}\left(K_{p}\right)$ and the restricted linear group $\operatorname{Gl}\left(K_{p}\right)$.

Let $\Gamma_{p}$ be the formal commutative group scheme representing the contravariant functor on the category of $k$-schemes:

$$
S \rightsquigarrow\left[H^{0}\left(S, \varnothing_{S}\right) \otimes_{k} k(p)\right]((z))^{*} \quad([10, \text { Section 3.B }) .
$$

If $p$ is a rational point and $\Gamma$ is the formal scheme defined above, then $\Gamma_{p} \simeq \Gamma$.

It is clear that $\Sigma_{X}^{*} \subseteq K_{p}^{*} \simeq \Gamma_{p}(\operatorname{Spec} k) \subseteq \mathrm{Gl}\left(K_{p}\right)(\operatorname{Spec} k)$.

There exists a central extension of group schemes:

$$
1 \rightarrow \mathbb{G}_{m} \rightarrow \widetilde{\Gamma}_{p} \stackrel{\pi_{p}}{\longrightarrow} \Gamma_{p} \rightarrow 1
$$

which, considering rational points and restricting to $\Sigma_{X}^{*}$, induces a central extension of groups:

$$
1 \rightarrow k^{*} \rightarrow \widetilde{\Sigma_{X}^{*}} \stackrel{\pi_{p}}{\longrightarrow} \Sigma_{X}^{*} \rightarrow 1
$$

whose commutator pairing is $\{, \quad\}_{A_{p}}^{K_{p}}: \Sigma_{X}^{*} \times \Sigma_{X}^{*} \rightarrow k^{*}$.

According to the statements of [11], if $f, g \in \Sigma_{X}^{*}$, then

$$
\{f, g\}_{A_{p}}^{K_{p}}=N_{k(p) / k}\left(\frac{f^{v_{p}(g)}}{g^{v_{p}(f)}}(p)\right) \in k^{*},
$$

where $N_{k(p) / k}$ denotes the norm of the extension $k \hookrightarrow k(p)$, and $v_{p}$ is the discrete valuation on $\Sigma_{X}^{*}$ associated with the closed point $p$.

It is therefore possible to recover the tame symbol of an algebraic curve from this commutator, and its explicit expression is:

$$
\begin{aligned}
(f, g)_{p} & =(-1)^{\operatorname{deg}(p) \cdot v_{p}(f) \cdot v_{p}(g)}\{f, g\}_{A_{p}}^{K_{p}} \\
& =(-1)^{\operatorname{deg}(p) \cdot v_{p}(f) \cdot v_{p}(g)} N_{k(p) / k}\left(\frac{f^{v_{p}(g)}}{g^{v_{p}(f)}}(p)\right) .
\end{aligned}
$$

When $X$ is complete, the Weil reciprocity law shows that

$$
\prod_{p \in X}(f, g)_{p}=1 .
$$


Remark 2.3. With the notation of Remark 2.2. for each function $f \in \Sigma_{X}^{*}$ we can define the integer number:

$$
i\left(f, A_{p}\right)=\operatorname{dim}_{k}\left(A_{p} / A_{p} \cap f A_{p}\right)-\operatorname{dim}_{k}\left(f A_{p} / A_{p} \cap f A_{p}\right)=\operatorname{deg}(p) \cdot v_{p}(f) .
$$

If $U_{p}^{f}$ is a $k$-vector subspace of $K_{p}$ such that $f A_{p} \oplus U_{p}^{f}=K_{p}$, then $U_{p}^{f}$ is a rational point of the Grassmannian $\operatorname{Gr}\left(K_{p}\right)$. One thus has that $i\left(f, A_{p}\right)=i_{U_{p}^{f}}$, where $i_{U_{p}^{f}}$ is the index defined by the formula (2.1).

This construction of the central extension $\widetilde{\Gamma}$ can be generalized to more general loop groups.

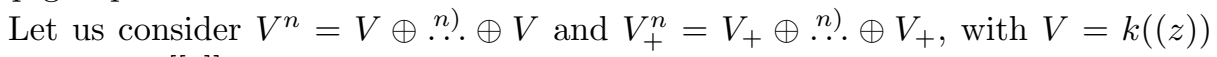
and $V_{+}=k[[z]]$. Following the same steps described above, we construct the vector Grassmannian $\operatorname{Gr}\left(V^{n}\right)$, the determinant bundle $\operatorname{Det}_{n}$, and the groups $\operatorname{Gl}\left(V^{n}\right)$ and $\widetilde{\mathrm{Gl}}\left(V^{n}\right)$. The loop group of $\operatorname{Gl}(n, k)$ is defined as the group scheme $\operatorname{LGl}(n)$, representing the functor of groups:

$$
S \rightsquigarrow \underline{\operatorname{LGl}}(n)(S)=\left\{\begin{array}{l}
\text { automorphisms of } V^{n} \hat{\otimes}_{k} H^{0}\left(S, \varnothing_{S}\right) \\
\text { as } H^{0}\left(S, \varnothing_{S}\right) \text {-modules }
\end{array}\right\} .
$$

$\mathrm{LGl}(n)$ is a subgroup of $\mathrm{Gl}\left(V^{n}\right)$, and therefore there exists a central extension of group functors:

$$
1 \rightarrow \mathbb{G}_{m} \rightarrow \widetilde{\operatorname{LGl}}(n) \rightarrow \operatorname{LGl}(n) \rightarrow 1
$$

\section{AdELIC CENTRAL EXTENSIONS AND GENERALIZED RECIPROCITY LAWS}

Let $X$ be a complete, smooth and connected curve over a perfect field $k$. Let $\Sigma_{X}$ be its function field, $\mathbb{A}_{X}$ the adele ring of $X$, and $\mathbb{A}_{X}^{+}$the subring

$$
\prod_{\substack{x \in X \\ x \text { closed }}} \widehat{\emptyset}_{x}
$$

of $\mathbb{A}_{X}$.

To the pair of $k$-vector spaces $\left(\mathbb{A}_{X}, \mathbb{A}_{X}^{+}\right)$we can associate a Grassmannian $\operatorname{Gr}_{X}=$ $\operatorname{Gr}\left(\mathbb{A}_{X}, \mathbb{A}_{X}^{+}\right)$, as described in the previous section. Let us denote by $\mathbb{D}$ the determinant bundle over $\mathrm{Gr}_{X}$. The group of ideles of $X, \mathbb{I}_{X}$, is a subgroup of $\operatorname{Gl}\left(\mathbb{A}_{X}\right)(\operatorname{Spec} k)$, the rational points of the restricted linear group, and then, applying the results of Subsection 2.B. there exists a canonical central extension of groups:

$$
1 \rightarrow k^{*} \rightarrow \widetilde{\mathbb{I}}_{X} \rightarrow \mathbb{I}_{X} \rightarrow 1 .
$$

In general, for each positive integer $n$, let us consider the $k$-vector spaces:

$$
\begin{aligned}
\mathbb{A}_{X}^{n} & \left.=\mathbb{A}_{X} \oplus \ldots n\right) \ldots \oplus \mathbb{A}_{X}, \\
\left(\mathbb{A}_{X}^{+}\right)^{n} & \left.=\mathbb{A}_{X}^{+} \oplus \ldots n\right) \ldots \oplus \mathbb{A}_{X}^{+},
\end{aligned}
$$

and let us denote by $\operatorname{Gr}_{X}^{n}$ the vector adelic Grassmannian $\operatorname{Gr}\left(\mathbb{A}_{X}^{n},\left(\mathbb{A}_{X}^{+}\right)^{n}\right)$ and by $\mathbb{D}_{n}$ its determinant bundle. The rank- $n$ restricted linear group $\operatorname{Gl}\left(\mathbb{A}_{X}^{n}\right)$ acts on $\operatorname{Gr}_{X}^{n}$, and we denote by $\operatorname{Gl}\left(n, \mathbb{A}_{X}\right)$ the adelic linear group of rank $n$ (that is, the group of invertible $n \times n$ matrices with entries in $\left.\mathbb{A}_{X}\right)$. Obviously, $\operatorname{Gl}\left(n, \mathbb{A}_{X}\right) \subset$ $\operatorname{Gl}\left(\mathbb{A}_{X}^{n}\right)(\operatorname{Spec} k)$, and one has a canonical central extension of groups:

$$
\left.1 \rightarrow k^{*} \rightarrow \widetilde{\operatorname{Gl}\left(n, \mathbb{A}_{X}\right.}\right) \stackrel{\pi}{\longrightarrow} \operatorname{Gl}\left(n, \mathbb{A}_{X}\right) \rightarrow 1 .
$$


If $g$ and $h$ are two commuting elements of $\operatorname{Gl}\left(n, \mathbb{A}_{X}\right)$ and $\widetilde{g}, \widetilde{h} \in \widetilde{\operatorname{Gl}\left(n, \mathbb{A}_{X}\right)}$ are elements such that $\pi(\widetilde{g})=g$ and $\pi(\widetilde{h})=h$, then one has a commutator pairing:

$$
\{g, h\}_{\left(\mathbb{A}_{X}^{+}\right)^{n}}^{\mathbb{A}^{n}}=\widetilde{g} \cdot \widetilde{h} \cdot \widetilde{g}^{-1} \cdot \widetilde{h}^{-1} \in k^{*} .
$$

Definition 3.1. The moduli space of rank $n$ vector bundles over $X$ with an infinitelevel trivialization is the set:

$$
\mathbb{M}_{n}^{\infty}(X)_{k}=\left\{\left(\mathcal{E}, \Phi^{\infty}\right)\right\} / \text { Isomorphisms },
$$

where $\mathcal{E}$ is a locally-free sheaf of $\operatorname{rank} n$ over $X$ and $\Phi^{\infty}$ an infinite-level trivialization of $\mathcal{E}$ :

$$
\left.\Phi^{\infty}:{\underset{\lim }{D}}_{\widehat{\mathcal{E}}_{D}} \stackrel{\sim}{\longrightarrow}\left(\mathbb{A}_{X}^{+}\right)^{n}=\mathbb{A}_{X}^{+} \oplus \ldots, \ldots\right) \oplus \mathbb{A}_{X}^{+}
$$

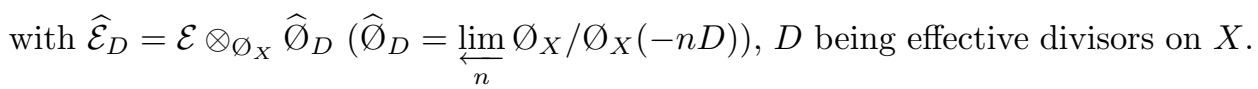

One can easily define the moduli functor $\mathbb{M}_{n}^{\infty}(X)$ of rank $n$ vector bundles over $X$ with infinite-level trivialization in such a way that $\mathbb{M}_{n}^{\infty}(X)_{k}$ are the points of $\mathbb{M}_{n}^{\infty}(X)$ with values in Spec $k$. See [1] for details in the case of trivialization at a point.

Proposition 3.2. There exists a Krichever map

$$
\mathrm{Kr}: \mathbb{M}_{n}^{\infty}(X)_{k} \longrightarrow \operatorname{Gr}_{X}^{n}(\operatorname{Spec} k)
$$

defined by: $\left.\operatorname{Kr}\left(\mathcal{E}, \Phi^{\infty}\right)=\Phi^{\infty} \underset{D}{[\lim } H^{0}(X-D, \mathcal{E})\right], D$ being the set of effective divisors on $X$.

Proof. This is the same as in ([1, Theorem 3.9).

Remark 3.3. $\operatorname{Kr}\left[\mathbb{M}_{n}^{\infty}(X)_{k}\right]$, as a subset of $\operatorname{Gr}_{X}^{n}(\operatorname{Spec} k)$, is stable under the action of $\operatorname{Gl}\left(n, \mathbb{A}_{X}\right)$. This follows from the well-known description of the moduli space of vector bundles in terms of $\operatorname{Gl}\left(n, \mathbb{A}_{X}\right)([6],[15])$.

Let us consider the diagonal immersion:

$$
\delta: \operatorname{Gl}\left(n, \Sigma_{X}\right) \hookrightarrow \operatorname{Gl}\left(n, \mathbb{A}_{X}\right)
$$

induced by the diagonal immersion $\Sigma_{X}^{*} \hookrightarrow \mathbb{I}_{X} \subset \mathbb{A}_{X}$. This immersion induces an action of $\operatorname{Gl}\left(n, \Sigma_{X}\right)$ on $\operatorname{Gr}_{X}^{n}(\operatorname{Spec} k)$.

Proposition 3.4. The points of $\operatorname{Kr}\left[\mathbb{M}_{n}^{\infty}(X)_{k}\right]$ are invariant under the action of $\operatorname{Gl}\left(n, \Sigma_{X}\right)$.

Proof. This statement is a reinterpretation of the usual description of the moduli stack of bundles over $X$ as a quotient of the loop group (see for example [6], 4.1.9).

This can be proved directly by observing that given an element $g \in \operatorname{Gl}\left(n, \Sigma_{X}\right)$, the image of the vector subspace $U=H^{0}(X-D, \mathcal{E}) \subset k((z))^{n}$ ( $\mathcal{E}$ again being a locally free sheaf of rank $n$ over $X)$ under the action of $g$ is a subspace of $H^{0}\left(X-\left(D+D_{g}\right), \mathcal{E}\right), D_{g}$ being the set of poles of the matrix $g$. Of course, this statement is only true if $X$ is a complete curve.

Theorem 3.5 (Abstract reciprocity law). The central extension

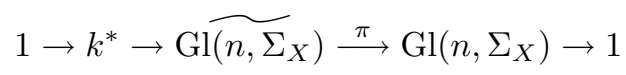


induced, through $\delta: \operatorname{Gl}\left(n, \Sigma_{X}\right) \hookrightarrow \operatorname{Gl}\left(n, \mathbb{A}_{X}\right)$, by the central extension (3.2), is trivial.

Proof. Let $\left[X_{n}\right]=\left(\varnothing_{X}^{n}, I d\right)$ be the canonical point of $\mathbb{M}_{n}^{\infty}(X)_{k}$ and let $\mathbb{D}\left[X_{n}\right]$ be the fibre of the determinant bundle over the point $\left[X_{n}\right]$. From the invariance of $\left[X_{n}\right]$ under the action of $\operatorname{Gl}\left(n, \Sigma_{X}\right)$ it follows that $\widetilde{\operatorname{Gl}\left(n, \Sigma_{X}\right)}$ acts on the fibre $\mathbb{D}\left[X_{n}\right]$, which is a $k$-vector space of dimension one. Thus, the character of this representation is a homomorphism of groups:

$$
\mu: \operatorname{Gl} \widetilde{\left(n, \Sigma_{X}\right)} \rightarrow k^{*} .
$$

Let $\left.\sigma: \operatorname{Gl}\left(n, \Sigma_{X}\right) \longrightarrow \operatorname{Gl} \widetilde{\left(n, \Sigma_{X}\right.}\right)$ be a section (in general not a group homomor-

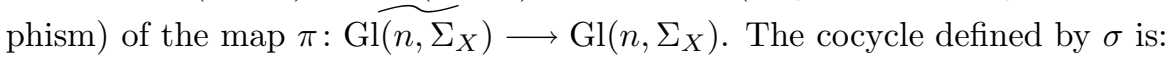

$$
\begin{aligned}
a: \operatorname{Gl}\left(n, \Sigma_{X}\right) \times \operatorname{Gl}\left(n, \Sigma_{X}\right) & \longrightarrow k^{*} \\
(g, h) & \longmapsto a(g, h):=\sigma(g) \sigma(h) \sigma(g h)^{-1} .
\end{aligned}
$$

Let us define $\bar{\mu}: \operatorname{Gl}\left(n, \Sigma_{X}\right) \rightarrow k^{*}$ as follows:

$$
\bar{\mu}(g)=\mu(\sigma(g)) .
$$

Applying $\mu$ to the identity defining the cocycle $a$, one has

$$
\bar{\mu}(g) \cdot \bar{\mu}(h)=a(g, h) \cdot \bar{\mu}(g h) .
$$

Let us now define another section $\widetilde{\sigma}$ :

$$
\begin{aligned}
\tilde{\sigma}: \operatorname{Gl}\left(n, \Sigma_{X}\right) & \longrightarrow \widetilde{\operatorname{Gl}\left(\tilde{n, \Sigma_{X}}\right)} \\
g & \longmapsto \widetilde{\sigma}(g)=\bar{\mu}(g)^{-1} \sigma(g) .
\end{aligned}
$$

Bearing in mind the identity (3.3), it can be easily proved that:

$$
\widetilde{a}(g, h):=\widetilde{\sigma}(g) \widetilde{\sigma}(h) \widetilde{\sigma}(g h)^{-1}=1,
$$

and therefore $\widetilde{\sigma}$ is a homomorphism of groups.

Corollary 3.6. Let $g$ and $h$ be two commuting elements of $\operatorname{Gl}\left(n, \Sigma_{X}\right)$ and let $\widetilde{g}, \widetilde{h} \in \operatorname{Gl}\left(n, \Sigma_{X}\right)$ be elements such that $\pi(\widetilde{g})=g$ and $\pi(\widetilde{h})=h$. One has

$$
\{g, h\}_{\left(\mathbb{A}_{X}^{+}\right)^{n}}^{\mathbb{A}_{n}^{n}}=\widetilde{g} \cdot \widetilde{h} \cdot \widetilde{g}^{-1} \cdot \widetilde{h}^{-1}=1 .
$$

Proof. This is a trivial consequence of Theorem 3.5 .

Remark 3.7 (Reciprocity law for the tame symbol). Let us show that the usual reciprocity law for the tame symbol can be recovered from Theorem 3.5

Let $X$ again be a complete, smooth and connected curve over a perfect field $k$, and let $p \in X$ be a closed point on it. Let us denote again by $A_{p}=\hat{\emptyset}_{p}$ the completion of the local ring $\varnothing_{X, p}$ and by $K_{p}=\left(\hat{\emptyset}_{p}\right)_{0}$ its field of fractions.

Let $\Gamma_{p}$ be the formal commutative scheme referred to in Remark 2.2

One has the following central extensions:

$$
\begin{aligned}
1 \rightarrow k^{*} & \rightarrow \widetilde{\Gamma}_{p}(\operatorname{Spec} k) \stackrel{\pi_{p}}{\longrightarrow} \Gamma_{p}(\operatorname{Spec} k) \rightarrow 1, \\
1 & \rightarrow k^{*} \rightarrow \widetilde{\mathbb{I}}_{X} \stackrel{\pi}{\longrightarrow} \mathbb{I}_{X} \rightarrow 1, \\
1 & \rightarrow k^{*} \rightarrow \widetilde{\Sigma_{X}^{*}} \stackrel{\pi}{\longrightarrow} \Sigma_{X}^{*} \rightarrow 1,
\end{aligned}
$$


and there exists the commutative diagram:

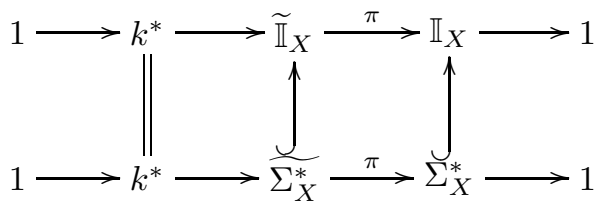

relating the central extensions (3.5) and (3.6).

By Theorem 3.5, the extension (3.6) is trivial. Moreover, the groups $\Gamma_{p}(\operatorname{Spec} k)$ and $\mathbb{I}_{X}$ define pairings:

$$
\begin{aligned}
\{\quad\}_{A_{p}}^{K_{p}}: \Gamma_{p}(\operatorname{Spec} k) \times \Gamma_{p}(\operatorname{Spec} k) & \longrightarrow k^{*} \\
(a, b) & \longmapsto\{a, b\}_{A_{p}}^{K_{p}}=\widetilde{a} \cdot \widetilde{b} \cdot \widetilde{a}^{-1} \cdot \widetilde{b}^{-1},
\end{aligned}
$$

with $\pi_{p}(\widetilde{a})=a, \pi_{p}(\widetilde{b})=b$, and

$$
\begin{aligned}
\{, \quad\}_{\mathbb{A}_{X}^{+}}^{\mathbb{A}_{X}}: \mathbb{I}_{X} \times \mathbb{I}_{X} & \longrightarrow k^{*} \\
(f, g) & \longmapsto\{f, g\}_{\mathbb{A}_{X}^{+}}^{\mathbb{A}_{X}}=\tilde{f} \cdot \widetilde{g} \cdot \tilde{f}^{-1} \cdot \widetilde{g}^{-1},
\end{aligned}
$$

where $\pi(\widetilde{f})=f$ and $\pi(\widetilde{g})=g$.

Via the natural immersion $\Sigma_{X}^{*} \hookrightarrow K_{p}^{*}=\Gamma_{p}(\operatorname{Spec} k)$, we have a pairing $\{\quad, \quad\}_{A_{p}}^{K_{p}}$ : $\Sigma_{X}^{*} \times \Sigma_{X}^{*} \rightarrow k^{*}$ for each closed point $p \in X$.

The triviality of (3.6) implies that given $f, g \in \Sigma_{X}^{*}$ one has

$$
\{f, g\}_{\mathbb{A}_{X}^{+}}^{\mathbb{A}_{X}}=1 .
$$

We shall show that the identity (3.7) is precisely the reciprocity law. Let us compute the restriction of the pairing $\{, \quad\}_{\mathbb{A}_{X}^{+}}^{\mathbb{A}_{X}}$ to $\Sigma_{X}^{*} \times \Sigma_{X}^{*}$ in terms of the pairings $\{, \quad\}_{A_{p}}^{K_{p}}$.

One can describe $\widetilde{\mathbb{I}}_{X}$ as in [1]:

$$
\widetilde{\mathbb{I}}_{X}=\left\{\begin{array}{c}
(f, s) \text { such that } f \in \mathbb{I}_{X} \text { and } \\
0 \neq s \in \Lambda\left(\mathbb{A}_{X}^{+} /\left[\mathbb{A}_{X}^{+} \cap f \mathbb{A}_{X}^{+}\right]\right) \otimes_{k} \Lambda\left(f \mathbb{A}_{X}^{+} /\left[\mathbb{A}_{X}^{+} \cap f \mathbb{A}_{X}^{+}\right]\right)^{*}
\end{array}\right\},
$$

with $f=\left(f_{x}\right)_{x \in X}$, and $f_{x_{i}} \notin A_{x_{i}}^{*}$ for a finite number of points $\left\{x_{i}\right\}$.

It is clear that

$$
\begin{aligned}
\mathbb{A}_{X}^{+} /\left[\mathbb{A}_{X}^{+} \cap f \mathbb{A}_{X}^{+}\right] & =\bigoplus_{x_{i} \in X} A_{x_{i}} /\left[A_{x_{i}} \cap f_{x_{i}} A_{x_{i}}\right], \\
f \mathbb{A}_{X}^{+} /\left[\mathbb{A}_{X}^{+} \cap f \mathbb{A}_{X}^{+}\right] & =\bigoplus_{x_{i} \in X} f_{x_{i}} A_{x_{i}} /\left[A_{x_{i}} \cap f_{x_{i}} A_{x_{i}}\right] .
\end{aligned}
$$

Given two elements $f=\left(f_{x}\right)_{x \in X}$ and $g=\left(g_{x}\right)_{x \in X}$ of $\Sigma_{X}^{*} \subseteq \mathbb{I}_{X}$, let $\left\{x_{i}\right\}$ be the finite set of points such that $f_{x_{i}} \notin A_{x_{i}}^{*}$, and let $\left\{y_{j}\right\}$ be the finite set of points such that $g_{y_{j}} \notin A_{y_{j}}^{*}$. Let us denote $D_{f g}=\left\{x_{i}\right\} \cup\left\{y_{j}\right\}$ : this is a finite set of closed points 
of $X$ and it follows from the statements of [1] that

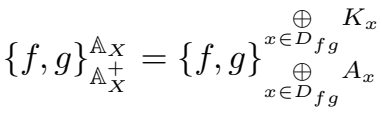

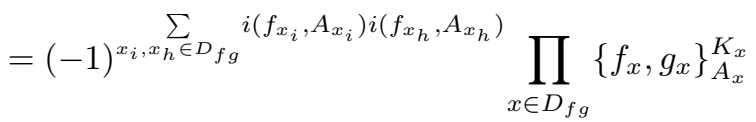

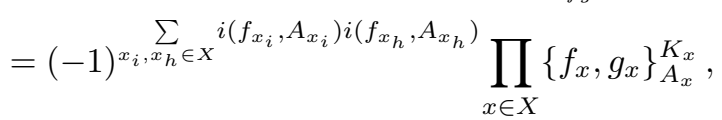

where $\operatorname{deg}(x)=\operatorname{dim}_{k} k(x)$, and $i\left(f_{x}, A_{x}\right)=\operatorname{deg}(x) v_{x}\left(f_{x}\right)$. Applying this computation to the case $f, g \in \Sigma_{X}^{*}$ one concludes the proof of the reciprocity law.

Remark 3.8 (Reciprocity laws associated with finite coverings). Let us consider a finite covering $\pi: Y \rightarrow X$ of a complete, smooth and connected curve over a perfect field $k$. Let $n$ be the degree of $\pi . \pi_{*} \varnothing_{Y}$ is a locally free sheaf of $\varnothing_{X \text {-modules of }}$ rank $n$. Let $\Phi^{\infty}$ be an infinite-level trivialization of $\pi_{*} \emptyset_{Y}$, that is,

$$
\Phi^{\infty}: \varlimsup_{D}\left(\widehat{\pi_{*} \varnothing_{Y}}\right)_{D} \stackrel{\sim}{\longrightarrow} \mathbb{A}_{X}^{n}
$$

Note that an infinite-level trivialization of the sheaf $\pi_{*} \varnothing_{Y}$ is equivalent to giving a structure of an $\mathbb{A}_{X}$-algebra on $\mathbb{A}_{X}^{n}$ with certain conditions. The trivialization $\Phi^{\infty}$ induces an inclusion of groups:

$$
\Sigma_{Y}^{*} \subset \operatorname{Gl}\left(n, \mathbb{A}_{X}\right),
$$

where $\Sigma_{Y}$ is the function field of $Y$. Therefore, the extension (3.2) induces a central extension:

$$
1 \rightarrow k^{*} \rightarrow \widetilde{\Sigma_{Y}^{*}} \stackrel{\pi}{\longrightarrow} \Sigma_{Y}^{*} \rightarrow 1
$$

Let $U=\operatorname{Kr}\left(\pi_{*} \varnothing_{Y}, \Phi^{\infty}\right) \in \operatorname{Gr}\left(\mathbb{A}_{X}^{n}\right)$ be the point of the adelic Grassmannian defined by $U$. Obviously $U$ is invariant under the action of $\Sigma_{Y}^{*}$, and hence the same arguments used in the proof of Theorem 3.5 prove that the extension (3.8) is trivial. Since $\Sigma_{Y}^{*}$ is commutative, one has that

$$
\{g, h\}_{\left(\mathbb{A}_{X}^{+}\right)^{n}}^{\mathbb{A}^{n}}=\widetilde{g} \widetilde{h} \widetilde{g}^{-1} \widetilde{h}^{-1}=1
$$

for all $g, h \in \Sigma_{Y}^{*}$.

In some sense, the reciprocity law (3.9) together with some maximality condition on $\operatorname{Gl}\left(n, \mathbb{A}_{X}\right)$ could be used to characterize finite coverings $Y \stackrel{\pi}{\longrightarrow} X$. One problem directly related to a hypothetical non-abelian class field theory should be the characterization of Galois coverings $Y \stackrel{\pi}{\longrightarrow} X$ and the construction, in terms of $\operatorname{Gl}\left(n, \mathbb{A}_{X}\right)$, of its Galois group.

Remark 3.9 (The non-abelian case). Keeping the assumptions about $X$ of Remark 3.7, let $G$ be a reductive algebraic group over $k$ and let us assume we have an immersion $G \subset \operatorname{Gl}(n, k)$. For each closed point $x \in X$, let us denote by $G_{x}$ the loop group over $k(x), G_{x} \subset \operatorname{LGl}(n)_{k(x)}$, where $\operatorname{LGl}(n)_{k(x)}$ is the group scheme representing the functor of groups:

$$
S \rightsquigarrow \underline{\operatorname{LGl}}(n)_{k(x)}(S)=\underline{\operatorname{LGl}}(n)\left(S \times_{\operatorname{Spec} k} \operatorname{Spec} k(x)\right) .
$$


Let $G_{\mathbb{A}_{X}}$ be the adelic group $\left(G_{\mathbb{A}_{X}} \subset \operatorname{Gl}\left(n, \mathbb{A}_{X}\right)\right)$, and let $G_{\Sigma_{X}}$ be the group over $\Sigma_{X}\left(G_{\Sigma_{X}} \subset \operatorname{Gl}\left(n, \Sigma_{X}\right)\right)$. The above results imply the existence of the following central extensions:

$$
\begin{gathered}
1 \rightarrow \mathbb{G}_{m} \rightarrow \widetilde{G}_{x} \longrightarrow G_{x} \rightarrow 1, \\
1 \rightarrow k^{*} \rightarrow \widetilde{G}_{\mathbb{A}_{X}} \longrightarrow G_{\mathbb{A}_{X}} \rightarrow 1, \\
1 \rightarrow k^{*} \rightarrow \widetilde{G_{\Sigma_{X}}} \longrightarrow G_{\Sigma_{X}} \rightarrow 1,
\end{gathered}
$$

where the extension (3.10) is induced by the morphism of the functor of groups

$$
\underline{N_{k(x) / k}}: \mathbb{G}_{m, k(x)}(S)=\left[H^{0}\left(S, \varnothing_{S}\right) \otimes_{k} k(x)\right]^{*} \longrightarrow \mathbb{G}_{m}(S)=H^{0}\left(S, \varnothing_{S}\right)^{*},
$$

and there again exists a commutative diagram:

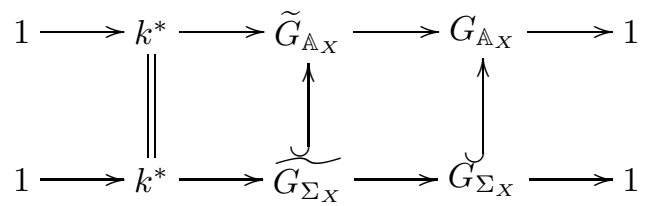

relating the central extensions (3.11) and (3.12).

From Theorem 3.5 it follows that (3.12) is a trivial central extension.

We have two ways to translate the triviality of the extension (3.12) into explicit identities that can be understood as non-abelian analogues of the reciprocity law. The first consists in applying Corollary 3.6 and explicitly computing the identity of that corollary in terms of the commutators of the groups $\widetilde{G}_{x}$. This approach will be explored in the next section in the case $n=2$.

The second approach consists in imposing the condition of triviality of the sequence (3.12) in terms of cocycles associated with some section of $\widetilde{G}_{\mathbb{A}_{X}} \rightarrow G_{\mathbb{A}_{X}}$ and computing these cocycles in terms of the cocycles of the local extensions (3.10). This approach will be developed in another paper.

Remark 3.10. Here we have used the theory of the Sato Grassmannian and determinant bundles to construct the central extensions. Of course, one can construct these extensions using a more elementary language, as is done for example in 3 . The reason for using these more sophisticated techniques is that in this way the results can be generalized to the case of arithmetic curves and, in general, curves defined over a ring $R$. We hope to develop this theory over arbitrary Noetherian rings elsewhere.

\section{Reciprocity LaWs on $\operatorname{Gl}\left(2, \Sigma_{X}\right)$}

This final section is devoted to applying the above theory to study reciprocity laws on the group $\operatorname{Gl}\left(2, \Sigma_{X}\right)$, where $\Sigma_{X}$ is again the function field of a smooth and connected curve over a perfect field $k$. Keeping the previous notation, we set $A_{p}=\hat{\emptyset}_{p}$ and $K_{p}=\left(\hat{\emptyset}_{p}\right)_{0}$.

Let us now consider $V_{2}^{+}=A_{p} \oplus A_{p}=A_{p}^{2}$ and $V_{2}=K_{p} \oplus K_{p}=K_{p}^{2}$. It is clear that $\operatorname{Gl}\left(2, \Sigma_{X}\right) \subseteq \operatorname{Gl}\left(K_{p}^{2}\right)(\operatorname{Spec} k)$, and hence the central extension of the functor of groups (2.2) induces a central extension of groups:

$$
1 \rightarrow k^{*} \longrightarrow \widetilde{\mathrm{Gl}}\left(2, \Sigma_{X}\right) \longrightarrow \mathrm{Gl}\left(2, \Sigma_{X}\right) \rightarrow 1
$$


whose commutator is denoted by $\{\varphi, \phi\}_{A_{p}^{2}}^{K_{2}^{2}}$ for all $\varphi, \phi \in \operatorname{Gl}\left(2, \Sigma_{X}\right)$, such that $\varphi \cdot \tau=\tau \cdot \varphi$. Similar to Remark 2.3, for each matrix $\varphi \in \operatorname{Gl}\left(2, \Sigma_{X}\right)$, one has the index

$$
i\left(\varphi, A_{p}^{2}\right)=\operatorname{dim}_{k}\left(A_{p}^{2} / A_{p}^{2} \cap \varphi A_{p}^{2}\right)-\operatorname{dim}_{k}\left(\varphi A_{p}^{2} / A_{p}^{2} \cap \varphi A_{p}^{2}\right) .
$$

Lemma 4.1. If $\varphi \in \operatorname{Gl}\left(2, \Sigma_{X}\right)$, then

$$
i\left(\varphi, A_{p}^{2}\right)=\operatorname{deg}(p) \cdot v_{p}(\operatorname{det} \varphi) .
$$

Proof. Bearing in mind the properties of the index, from the decomposition

$$
\varphi=\left(\begin{array}{cc}
\operatorname{det} \varphi & 0 \\
0 & 1
\end{array}\right) \cdot \widetilde{\varphi}
$$

where $\operatorname{det} \widetilde{\varphi}=1$, one has that $i\left(\varphi, A_{p}^{2}\right)=i\left(\left(\begin{array}{cc}\operatorname{det} \varphi & 0 \\ 0 & 1\end{array}\right), A_{p}^{2}\right)+i\left(\widetilde{\varphi}, A_{p}^{2}\right)$, with

$$
i\left(\left(\begin{array}{cc}
\operatorname{det} \varphi & 0 \\
0 & 1
\end{array}\right), A_{p}^{2}\right)=i\left(\operatorname{det} \varphi, A_{p}\right)=\operatorname{deg}(p) \cdot v_{p}(\operatorname{det} \varphi) .
$$

Thus, to prove the claim of the lemma it is sufficient to see that $i\left(\widetilde{\varphi}, A_{p}^{2}\right)=0$.

Moreover, it also follows from the additivity of the index that $i\left(\widetilde{\varphi}, A_{p}^{2}\right)=$ $i\left(J_{\widetilde{\varphi}}, A_{p}^{2}\right)$, where $J_{\widetilde{\varphi}}$ is the Jordan matrix associated with $\widetilde{\varphi}$.

Since $J_{\widetilde{\varphi}} \in\left\{\left(\begin{array}{cc} \pm 1 & 0 \\ 1 & \pm 1\end{array}\right),\left(\begin{array}{cc}0 & -1 \\ 1 & \operatorname{tr} \widetilde{\varphi}\end{array}\right),\left(\begin{array}{cc}g & 0 \\ 0 & g^{-1}\end{array}\right)\right\}$, one has that

$$
\operatorname{dim}_{k}\left(A_{p}^{2} /\left[A_{p}^{2} \cap J_{\widetilde{\varphi}} A_{p}^{2}\right]\right)=\operatorname{dim}_{k}\left(A_{p}^{2} /\left[A_{p}^{2} \cap J_{\widetilde{\varphi}}^{-1} A_{p}^{2}\right]\right),
$$

and we conclude that $i\left(\widetilde{\varphi}, A_{p}^{2}\right)=i\left(J_{\widetilde{\varphi}}, A_{p}^{2}\right)=0$.

Theorem 4.2 (Reciprocity Law on $\mathrm{Gl}\left(2, \Sigma_{X}\right)$ ). If $X$ is a complete, smooth and connected curve, for all commuting elements $\tau, \varphi \in \operatorname{Gl}\left(2, \Sigma_{X}\right)$ one has that

$$
\prod_{p \in X}(-1)^{\operatorname{deg}(p) v_{p}(\operatorname{det} \tau) v_{p}(\operatorname{det} \varphi)}\{\tau, \varphi\}_{A_{p}^{2}}^{K_{p}^{2}}=1 .
$$

Proof. If $\{\tau, \varphi\}_{\left(\mathbb{A}_{X}^{+}\right)^{2}}^{\mathbb{A}_{X}^{2}}$ is the commutator of the adelic central extension induced by $\delta: \operatorname{Gl}\left(2, \Sigma_{X}\right) \hookrightarrow \operatorname{Gl}\left(2, \mathbb{A}_{X}\right)$, it follows from Theorem 3.5 and Corollary 3.6 that $\{\tau, \varphi\}_{\left(\mathbb{A}_{X}^{+}\right)^{2}}^{\mathbb{A}_{X}^{2}}=1$ for all commuting elements $\tau, \varphi \in \operatorname{Gl}\left(2, \Sigma_{X}\right)$.

With similar arguments to Remark 3.7 one can see that

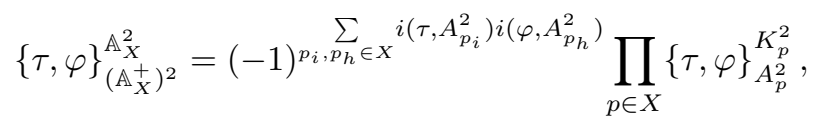

and the claim is a direct consequence of Lemma 4.1 because

$$
\sum_{p \in X} i\left(\tau, A_{p}^{2}\right)=\sum_{p \in X} i\left(\varphi, A_{p}^{2}\right)=0
$$

when $X$ is complete.

Remark 4.3 (Generalized tame symbol on $\operatorname{Gl}\left(n, \Sigma_{X}\right)$ ). For two commuting elements $\tau, \varphi \in \operatorname{Gl}\left(2, \Sigma_{X}\right)$, we can define a generalization of the tame symbol of an algebraic curve by the expression:

$$
(\tau, \varphi)_{2, p}=(-1)^{i\left(\tau, A_{p}^{2}\right) \cdot i\left(\varphi, A_{p}^{2}\right)}\{\tau, \varphi\}_{A_{p}^{2}}^{K_{p}^{2}} \in k^{*},
$$


and Theorem 4.2 is a generalization of the Weil reciprocity law because it shows that

$$
\prod_{p \in X}(\tau, \varphi)_{2, p}=1
$$

Similarly, it is possible to define the symbol $\left(\sigma, \sigma^{\prime}\right)_{n, p}$ for commuting matrices $\sigma, \sigma^{\prime} \in \operatorname{Gl}\left(n, \Sigma_{X}\right)$.

We conjecture that the result of Lemma 4.1 is true for $\operatorname{Gl}\left(n, \Sigma_{X}\right)$ by considering

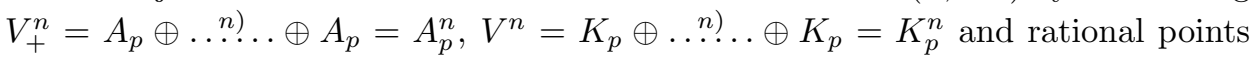
of the group scheme $\mathrm{Gl}\left(K_{p}^{n}\right)$. Solving this conjecture, the following reciprocity law arises directly:

$$
\prod_{p \in X}\left(\sigma, \sigma^{\prime}\right)_{n, p}=1
$$

Recall now from [3] that the commutator $\{\quad, \quad\}_{V_{+}}^{V}$ satisfies the following properties:

- Suppose $V$ is equipped with a direct sum decomposition $V=V_{0} \oplus V_{1}$. Put $V_{i+}:=V_{i} \cap V_{+}$for $i=0,1$ and assume that $V_{+}=V_{0+} \oplus V_{1+}$. Let commuting elements $\tau_{0}, \tau_{1} \in \operatorname{Gl}\left(V, V_{+}\right)$be given such that

$$
\left.\tau_{i}\right|_{V_{0}} \in \mathrm{Gl}\left(V_{0}, V_{0+}\right),\left.\quad \tau_{i}\right|_{V_{1}}=1
$$

for $i=0,1$. Then we have

$$
\left\{\left.\tau_{0}\right|_{V_{0}},\left.\tau_{1}\right|_{V_{0}}\right\}_{V_{0+}}^{V_{0}}=\left\{\tau_{0}, \tau_{1}\right\}_{V_{+}}^{V} .
$$

- Again suppose $V$ is equipped with a direct sum decomposition $V=V_{0} \oplus V_{1}$, put $V_{i+}:=V_{i} \cap V_{+}$for $i=0,1$ and assume that $V_{+}=V_{0+} \oplus V_{1+}$. Let $\tau_{0}, \tau_{1} \in \operatorname{Gl}\left(V, V_{+}\right)$be given such that

$$
\left.\tau_{i}\right|_{V_{i}} \in \operatorname{Gl}\left(V_{i}, V_{i+}\right),\left.\quad \tau_{i}\right|_{V_{1-i}}=1
$$

for $i=0,1$ (necessarily $\tau_{0}$ and $\tau_{1}$ commute). Then we have

$$
\left\{\tau_{0}, \tau_{1}\right\}_{V_{+}}^{V}=(-1)^{\nu_{0} \nu_{1}}
$$

where

$$
\nu_{i}:=i\left(\left.\tau_{i}\right|_{V_{i}}, V_{i+}\right)=i\left(\tau_{i}, V_{+}\right)
$$

for $i=0,1$.

Example 1. If we consider the commuting elements $\tau, \varphi \in \operatorname{Gl}\left(2, \Sigma_{X}\right)$, where

$$
\tau=\left(\begin{array}{cc}
f_{1} & 0 \\
0 & f_{2}
\end{array}\right) \quad \text { and } \quad \varphi=\left(\begin{array}{cc}
g_{1} & 0 \\
0 & g_{2}
\end{array}\right),
$$

with $f_{i}, g_{j} \in \Sigma_{X}^{*}$, we have that

$$
\begin{aligned}
\{\tau, \varphi\}_{A_{p}^{2}}^{K_{p}^{2}} & =\left\{\left(\begin{array}{cc}
f_{1} & 0 \\
0 & 1
\end{array}\right),\left(\begin{array}{cc}
g_{1} & 0 \\
0 & 1
\end{array}\right)\right\}_{A_{p}^{2}}^{K_{2}^{2}} \cdot\left\{\left(\begin{array}{cc}
f_{1} & 0 \\
0 & 1
\end{array}\right),\left(\begin{array}{cc}
1 & 0 \\
0 & g_{2}
\end{array}\right)\right\}_{A_{p}^{2}}^{K_{2}^{2}} \\
& \cdot\left\{\left(\begin{array}{cc}
1 & 0 \\
0 & f_{2}
\end{array}\right),\left(\begin{array}{cc}
g_{1} & 0 \\
0 & 1
\end{array}\right)\right\}_{A_{p}^{2}}^{K_{2}^{2}} \cdot\left\{\left(\begin{array}{cc}
1 & 0 \\
0 & f_{2}
\end{array}\right),\left(\begin{array}{cc}
1 & 0 \\
0 & g_{2}
\end{array}\right)\right\}_{A_{p}^{2}}^{K_{2}^{2}} \\
& =(-1)^{\operatorname{deg}(p)\left[v_{p}\left(f_{1}\right) v_{p}\left(g_{2}\right)+v_{p}\left(f_{2}\right) v_{p}\left(g_{1}\right)\right]} \cdot\left\{f_{1}, g_{1}\right\}_{A_{p}}^{K_{p}} \cdot\left\{f_{2}, g_{2}\right\}_{A_{p}}^{K_{p}},
\end{aligned}
$$


and Lemma 4.1 shows that the reciprocity law is

$$
\begin{aligned}
\prod_{p \in X}(-1)^{\operatorname{deg}(p) v_{p}(\operatorname{det} \tau) v_{p}(\operatorname{det} \varphi)}\{\tau, \varphi\}_{A_{p}^{2}}^{K_{p}^{2}} & =\prod_{p \in X}(-1)^{\operatorname{deg}(p) v_{p}\left(f_{1} \cdot f_{2}\right) v_{p}\left(g_{1} \cdot g_{2}\right)}\{\tau, \varphi\}_{A_{p}^{2}}^{K_{2}^{2}} \\
& =\prod_{p \in X}\left(f_{1}, g_{1}\right)_{p} \cdot\left(f_{2}, g_{2}\right)_{p}=1,
\end{aligned}
$$

where $(,)_{p}$ is the tame symbol at the closed point $p \in X$. Therefore, in this case, the reciprocity law of the commutator $\{\tau, \varphi\}_{A_{p}^{2}}^{K_{p}^{2}}$ can be deduced from the Weil reciprocity law.

Example 2. Let us now consider the commuting elements

$$
\tau=\left(\begin{array}{ll}
0 & f \\
1 & g
\end{array}\right) \quad \text { and } \quad \varphi=\left(\begin{array}{cc}
1 & f \\
1 & 1+g
\end{array}\right)
$$

with $f, g \in \Sigma_{X}^{*} \cap A_{p}$; that is, $v_{p}(f)=\alpha \geq 0, v_{p}(g)=\beta \geq 0$ and $1+g-f \neq 0$. To simplify, we assume in this example that the ground field $k$ is algebraically closed.

Let $x$ and $y$ be two elements of $A_{p}$.

If $x=c_{x} f+r_{x}$, one has that $(x, y)=\left(y-c_{x} g\right) \cdot(0,1)+c_{x} \cdot(f, g)+\left(r_{x}, 0\right)$, and there exists an isomorphism of $k$-vector spaces

$$
A_{p}^{2} / \tau A_{p}^{2} \simeq A_{p} /\langle f\rangle .
$$

Also, setting $x-y=\bar{c}(f-g-1)+\bar{r}$, it follows from the equality $(x, y)=$ $(x-\bar{c} f)(1,1)+\bar{c}(f, 1+g)+(0, \bar{r})$ that

$$
A_{p}^{2} / \varphi A_{p}^{2} \simeq A_{p} /\langle f-g-1\rangle
$$

Moreover, since

$$
\tau \varphi=\varphi \tau=\left(\begin{array}{cc}
f & f(1+g) \\
1+g & f+g+g^{2}
\end{array}\right)
$$

by setting $x=c_{x} f+r_{x}$ and $y-c_{x}(1+g)=c_{\widetilde{y}}(f-g-1)+r_{\widetilde{y}}$, from the expression

$$
(x, y)=\left[c_{x}-c_{\widetilde{y}}(1+g)\right](f, 1+g)+c_{\widetilde{y}}\left(f(1+g), f+g+g^{2}\right)+\left(r_{x}, r_{\widetilde{y}}\right),
$$

we deduce that

$$
A_{p}^{2} / \varphi \tau A_{p}^{2} \simeq A_{p} /\langle f\rangle \oplus A_{p} /\langle f-g-1\rangle .
$$

Bearing in mind the statements of [4] and [11, we have that the group $\widetilde{\mathrm{Gl}}(V)(\mathrm{Spec} k)$ is defined as

$$
\widetilde{\mathrm{Gl}}(V)(\operatorname{Spec} k)=\left\{(f, s) \text { with } f \in \operatorname{Gl}(V)(\operatorname{Spec} k), s \in \operatorname{Det} \mathcal{C}_{f\left(V_{+}\right)}^{\bullet}, s \neq 0\right\},
$$

together with the multiplication $(f, s) \cdot\left(g, s^{\prime}\right)=\left(f g, s \cdot \bar{f}\left(s^{\prime}\right)\right)$, where $\operatorname{Det} \mathcal{C}_{f\left(V_{+}\right)}$is the $k$-vector space:

$$
\operatorname{Det} \mathcal{C}_{f\left(V_{+}\right)}:=\Lambda V_{+} /\left[V_{+} \cap f V_{+}\right] \otimes_{k} \Lambda\left(f V_{+} /\left[V_{+} \cap f V_{+}\right]\right)^{*},
$$

$\Lambda$ again being the maximal exterior power, and

$$
\bar{f}\left(s^{\prime}\right) \in \Lambda f V_{+} /\left[f V_{+} \cap f g V_{+}\right] \otimes_{k} \Lambda\left(f g V_{+} /\left[f V_{+} \cap f g V_{+}\right]\right)^{*}
$$

being determined by the $k$-morphisms

$$
\begin{gathered}
f: V_{+} /\left[V_{+} \cap g V_{+}\right] \longrightarrow f V_{+} /\left[f V_{+} \cap f g V_{+}\right], \\
f^{-1}: f g V_{+} /\left[f V_{+} \cap f g V_{+}\right] \longrightarrow g V_{+} /\left[V_{+} \cap g V_{+}\right] .
\end{gathered}
$$

Thus, $\{f, g\}_{V_{+}}^{V}=\left[(f, s) \cdot\left(g, s^{\prime}\right)\right] \cdot\left[\left(g, s^{\prime}\right) \cdot(f, s)\right]^{-1} \in k^{*}$. 
Hence, fixing an isomorphism $A_{p} \simeq k[[t]]$, we can consider the elements $\left\{\widetilde{v}_{1}=\right.$ $\left.(1,0), \widetilde{v}_{2}=(t, 0), \ldots, \widetilde{v}_{\alpha}=\left(t^{\alpha-1}, 0\right)\right\} \subseteq A_{p}^{2} / \varphi \tau A_{p}^{2}$ such that their images in $A_{p}^{2} / \tau A_{p}^{2}$ determine a basis $\left\langle v_{i}\right\rangle$ of this $k$-vector space. Similarly, we have a basis $\left\langle w_{j}\right\rangle$ of $A_{p}^{2} / \varphi A_{p}^{2}$ consisting of the images of the elements $\left\{\widetilde{w}_{1}=(0,1), \widetilde{w}_{2}=(0, t), \ldots, \widetilde{w}_{\beta}=\right.$ $\left.\left(0, t^{\beta-1}\right)\right\} \subseteq A_{p}^{2} / \varphi \tau A_{p}^{2}$ in $A_{p}^{2} / \varphi A_{p}^{2}$ via the natural projection.

Then, $\operatorname{Det} \mathcal{C}_{\tau A_{p}^{2}}^{\bullet}=\Lambda A_{p}^{2} / \tau A_{p}^{2}=\left\langle v_{1} \wedge \cdots \wedge v_{\alpha}\right\rangle$ and $\operatorname{Det} \mathcal{C}_{\varphi A_{p}^{2}}=\Lambda A_{p}^{2} / \varphi A_{p}^{2}=$ $\left\langle w_{1} \wedge \cdots \wedge w_{\beta}\right\rangle$, and an easy computation shows that

$$
\bar{\tau}\left(w_{1} \wedge \cdots \wedge w_{\beta}\right)=\left(-\widetilde{w}_{1}\right) \wedge \cdots \wedge\left(-\widetilde{w}_{\beta}\right)=(-1)^{v(f-g-1)}\left(\widetilde{w}_{1} \wedge \cdots \wedge \widetilde{w}_{\beta}\right),
$$

and that

$$
\bar{\varphi}\left(v_{1} \wedge \cdots \wedge v_{\alpha}\right)=\left(\widetilde{v}_{1}+\widetilde{w}_{1}\right) \wedge \cdots \wedge\left(\widetilde{v}_{\alpha}+\widetilde{w}_{\alpha}\right)
$$

with $\widetilde{w}_{j}=0$ when $\beta<j \leq \alpha$.

Thus, denoting $s_{1}=v_{1} \wedge \cdots \wedge v_{\alpha}$ and $s_{2}=w_{1} \wedge \cdots \wedge w_{\beta}$, from the exact sequence of $k$-vector spaces

$$
0 \rightarrow \tau A_{p}^{2} / \tau \varphi A_{p}^{2} \rightarrow A_{p}^{2} / \tau \varphi A_{p}^{2} \rightarrow A_{p}^{2} / \tau A_{p}^{2} \rightarrow 0
$$

bearing in mind the explicit definition of the group law of $\widetilde{\mathrm{Gl}}(V)([4$, , 11]), one has that

$$
\begin{aligned}
\left(\tau, s_{1}\right) \cdot\left(\varphi, s_{2}\right) & =\left(\tau \varphi, s_{1} \cdot \bar{\tau}\left(s_{2}\right)\right) \\
& =\left(\tau \varphi,(-1)^{v_{p}(f-g-1)} \cdot \widetilde{w}_{1} \wedge \cdots \wedge \widetilde{w}_{\beta} \wedge \widetilde{v}_{1} \wedge \cdots \wedge \widetilde{v}_{\alpha}\right) .
\end{aligned}
$$

Analogously, from the exact sequence:

$$
0 \rightarrow \varphi A_{p}^{2} / \tau \varphi A_{p}^{2} \rightarrow A_{p}^{2} / \tau \varphi A_{p}^{2} \rightarrow A_{p}^{2} / \varphi A_{p}^{2} \rightarrow 0,
$$

we have that

$$
\begin{aligned}
\left(\varphi, s_{2}\right) \cdot\left(\tau, s_{1}\right) & =\left(\tau \varphi, s_{2} \cdot \bar{\varphi}\left(s_{1}\right)\right) \\
& =\left(\tau \varphi,\left(\widetilde{v}_{1}+\widetilde{w}_{1}\right) \wedge \cdots \wedge\left(\widetilde{v}_{\alpha}+\widetilde{w}_{\alpha}\right) \wedge \widetilde{w}_{1} \wedge \cdots \wedge \widetilde{w}_{\beta}\right) \\
& =\left(\tau \varphi,(-1)^{v_{p}(f) v_{p}(f-g-1)} \cdot \widetilde{w}_{1} \wedge \cdots \wedge \widetilde{w}_{\beta} \wedge \widetilde{v}_{1} \wedge \cdots \wedge \widetilde{v}_{\alpha}\right)
\end{aligned}
$$

Then,

$$
\{\tau, \varphi\}_{A_{p}^{2}}^{K_{p}^{2}}=(-1)^{v_{p}(f) v_{p}(f-g-1)+v_{p}(f-g-1)},
$$

and since $\operatorname{det} \tau=-f$ and $\operatorname{det} \varphi=1+g-f$, Theorem 4.2 shows that

$$
\prod_{p \in X}(-1)^{v_{p}(\operatorname{det} \varphi)}=1
$$

which is equivalent to the well-known formula $\sum_{p} v_{p}(\operatorname{det} \varphi)=0$ when $X$ is a complete curve over an algebraically closed field. Moreover, since $(-1)^{\operatorname{det} \tau \operatorname{det} \varphi}\{\tau, \varphi\}_{A_{p}^{2}}^{K_{2}^{2}}$ $=(-1, \operatorname{det} \varphi)_{p}$, the reciprocity law can also be deduced from the Weil reciprocity law in this example.

A remaining question is to characterize the commutator $\{\tau, p(\tau)\}_{A_{p}^{2}}^{K_{2}^{2}}$ for two commuting elements $\tau, p(\tau)$, where $\tau \in \operatorname{Gl}\left(2, \Sigma_{X}\right)$ and $p(x)$ is a polynomial with coefficients in $k$. 
Finally, regarding $\Sigma_{X}^{*}$ as a subgroup of $\operatorname{Gl}\left(2, \Sigma_{X}\right)$ by means of the diagonal embedding $f \mapsto \sigma_{f}:=\left(\begin{array}{ll}f & 0 \\ 0 & f\end{array}\right)$, one has that $\Sigma_{X}^{*}=Z\left(\operatorname{Gl}\left(2, \Sigma_{X}\right)\right)$ and, therefore, there exists a commutator map

$$
\{\quad, \quad\}_{A_{p}^{2}}^{K_{p}^{2}}: \Sigma_{X}^{*} \times \mathrm{Gl}\left(2, \Sigma_{X}\right) \rightarrow k^{*} .
$$

We shall compute the explicit expression of this commutator map.

Lemma 4.4. If $f \in \Sigma_{X}^{*}$, then $i\left(\sigma_{f}, A_{p}^{2}\right)=2 \operatorname{deg}(p) v_{p}(f)$.

Proof. The claim is a direct consequence of Lemma 4.1 .

Lemma 4.5. Setting

$$
J^{1}:=\left(\begin{array}{cc} 
\pm 1 & 0 \\
1 & \pm 1
\end{array}\right) \quad \text { and } \quad J^{2}:=\left(\begin{array}{cc}
0 & -1 \\
1 & h
\end{array}\right)
$$

with $h \in A_{p}$, one has that $\left\{\sigma_{f}, J^{1}\right\}_{A_{p}^{2}}^{K_{2}^{2}}=\left\{\sigma_{f}, J^{2}\right\}_{A_{p}^{2}}^{K_{2}^{2}}=1$ for all $f \in \Sigma_{X}^{*}$.

Proof. With the notation of Example 2, if $g \in \operatorname{Aut}_{k}\left(V_{+}\right)$, then $\operatorname{Det} \mathcal{C}_{g\left(V_{+}\right)}^{\bullet}=k$ is trivial, and $\bar{f}: \operatorname{Det}_{\mathcal{C}_{\left(V_{+}\right)}^{\bullet}} \rightarrow \operatorname{Det} \mathcal{C}_{g\left(V_{+}\right)}^{\bullet}$ is the identity on $k$ for all $f \in \operatorname{Gl}\left(V, V_{+}\right)$.

Let us assume that $v_{p}(f) \geq 1$. Then,

$$
A_{p}^{2} /\left[A_{p}^{2} \cap \sigma_{f} A_{p}^{2}\right] \simeq k(p)[[t]] / t^{n} \oplus k(p)[[t]] / t^{n},
$$

with $n=v_{p}(f)$. Let $\left\{e_{1}, \ldots, e_{d}\right\}$ be a basis of $k(p)$ as a $k$-vector space, $d=\operatorname{deg}(p)$, and let us consider the basis $\left\{e_{j} \cdot t^{i}\right\}_{i \in\{0, \ldots, n-1\}}^{j \in\{1, \ldots, d\}}$ of $k(p)[[t]] / t^{n}$ as a vector space over $k$.

Thus, $\left\{\left(e_{j} \cdot t^{i}, 0\right),\left(0, e_{j} \cdot t^{i}\right)\right\}_{i \in\{0, \ldots, n-1\}}^{j \in\{1, \ldots, d\}}$ is a basis of $k(p)[[t]] / t^{n} \oplus k(p)[[t]] / t^{n}$ as a $k$-vector space.

It is clear that $J^{i} \in \operatorname{Aut}_{k}\left(A_{p}^{2}\right)$ and, therefore, $\bar{\sigma}_{f}: \operatorname{Det} \mathcal{C}_{J^{i}\left(A_{p}^{2}\right)}^{\bullet} \rightarrow \operatorname{Det} \mathcal{C}_{J^{i}\left(A_{p}^{2}\right)}$ is the identity.

We set $\widetilde{J^{1}}$ and $\widetilde{J^{2}}$ to denote the $k$-automorphisms of $A_{p}^{2} /\left[A_{p}^{2} \cap \sigma_{f} A_{p}^{2}\right]$ induced by $J^{1}$ and $J^{2}$ respectively. We shall now compute $\operatorname{det} \widetilde{J^{1}}$ and $\operatorname{det} \widetilde{J^{2}}$ in order to determine $\bar{J}^{1}, \bar{J}^{2} \in \operatorname{Aut}_{k}\left(\Lambda A_{p}^{2} /\left[A_{p}^{2} \cap \sigma_{f} A_{p}^{2}\right]\right)$.

Bearing in mind that

$$
\begin{aligned}
& \widetilde{J^{1}}\left(e_{j} \cdot t^{i}, 0\right)=\left( \pm e_{j} \cdot t^{i}, e_{j} \cdot t^{i}\right), \\
& \widetilde{J^{1}}\left(0, e_{j} \cdot t^{i}\right)=\left(0, \pm e_{j} \cdot t^{i}\right),
\end{aligned}
$$

the automorphism $\widetilde{J^{1}}$ is determined by the matrix:

$$
\widetilde{J^{1}}=\left(\begin{array}{ccc}
A_{1} & & 0 \\
& \ddots & \\
0 & & A_{d}
\end{array}\right) \text { with } A_{j}=\left(\begin{array}{cccccccc} 
\pm 1 & & 0 & \vdots & & & \\
& \ddots & & \vdots & & 0 & \\
& & & \vdots & & & \\
0 & & \pm 1 & \vdots & & & \\
\ldots & \ldots & \ldots & \vdots & \ldots & \ldots & \ldots \\
1 & & 0 & \vdots & \pm 1 & & 0 \\
& \ddots & & \vdots & & \ddots & \\
& & & 1 & \vdots & 0 & & \pm 1
\end{array}\right) .
$$


Thus, det $\widetilde{J^{1}}=1$ and $\bar{J}^{1}(s)=s$ for all $s \in \operatorname{Det} \mathcal{C}_{\sigma_{f}}^{\bullet} A_{p}^{2}=\Lambda A_{p}^{2} /\left[A_{p}^{2} \cap \sigma_{f} A_{p}^{2}\right]$.

Moreover, since

$$
\begin{aligned}
& \widetilde{J^{2}}\left(e_{j} \cdot t^{i}, 0\right)=\left(0, e_{j} \cdot t^{i}\right) \\
& \widetilde{J^{2}}\left(0, e_{j} \cdot t^{i}\right)=\left(-e_{j} \cdot t^{i}, e_{j} \cdot\left[h t^{i} \bmod \cdot t^{n}\right]\right),
\end{aligned}
$$

$\widetilde{J^{2}} \in \operatorname{Aut}_{k}\left(A_{p}^{2} /\left[A_{p}^{2} \cap \sigma_{f} A_{p}^{2}\right]\right)$ is characterized by the matrix:

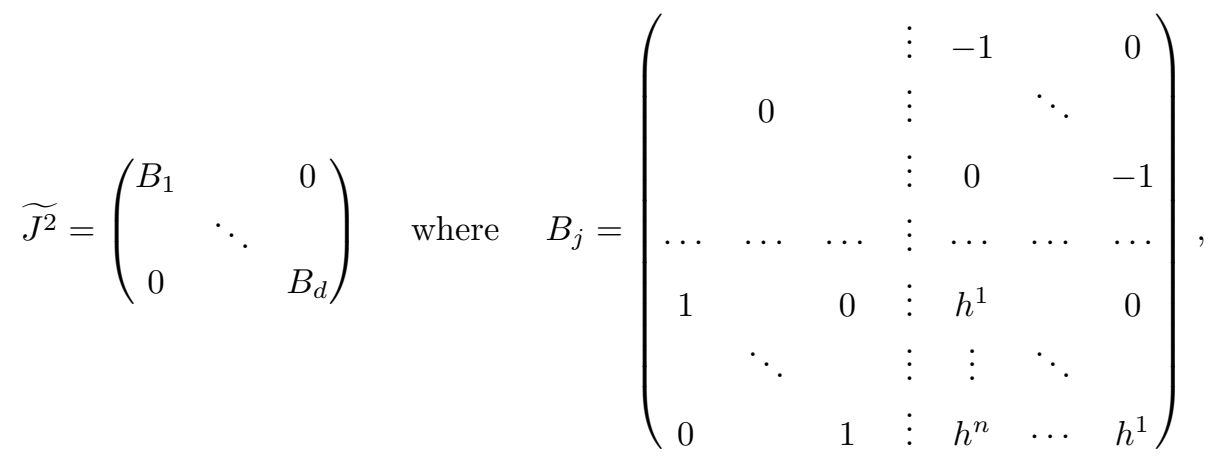

and, again, $\operatorname{det} \widetilde{J^{2}}=1$ and $\bar{J}^{2}(s)=s$ for all

$$
s \in \operatorname{Det} \mathcal{C}_{\sigma_{f} A_{p}^{2}}^{\bullet}=\Lambda A_{p}^{2} /\left[A_{p}^{2} \cap \sigma_{f} A_{p}^{2}\right] .
$$

Thus, when $v_{p}(f) \geq 1$, if $0 \neq s \in \operatorname{Det} \mathcal{C}_{\sigma_{f} A_{p}^{2}}$ and $0 \neq \lambda_{i} \in \operatorname{Det} \mathcal{C}_{J^{i} A_{p}^{2}}$, then

$$
\begin{aligned}
\left\{\sigma_{f}, J^{i}\right\}_{A_{p}^{2}}^{K_{2}^{2}} & =\left[\left(\sigma_{f}, s\right) \cdot\left(J^{i}, \lambda_{i}\right)\right] \cdot\left[\left(J^{i}, \lambda_{i}\right) \cdot\left(\sigma_{f}, s\right)\right]^{-1} \\
& =\left(\sigma_{f} \cdot J^{i}, s \cdot \overline{\sigma_{f}}\left(\lambda_{i}\right)\right) \cdot\left(J^{i} \sigma_{f}, \lambda_{i} \cdot \bar{J}^{i}(s)\right)^{-1} \\
& =\left(\sigma_{f} \cdot J^{i}, \lambda_{i} s\right) \cdot\left(\sigma_{f} \cdot J^{i}, \lambda_{i} s\right)^{-1}=1 .
\end{aligned}
$$

Bearing in mind that

$$
\left\{\operatorname{Id}, J^{i}\right\}_{A_{p}^{2}}^{K_{2}^{2}}=1=\left\{\sigma_{f}, J^{i}\right\}_{A_{p}^{2}}^{K_{2}^{2}}\left\{\sigma_{f^{-1}}, J^{i}\right\}_{A_{p}^{2}}^{K_{p}^{2}}
$$

we conclude the proof.

Proposition 4.6. For all $f \in \Sigma_{X}^{*}$ and $\varphi \in \operatorname{Gl}\left(2, \Sigma_{X}\right)$, one has that

$$
\left\{\sigma_{f}, \varphi\right\}_{A_{p}^{2}}^{K_{2}^{2}}=(-1)^{\operatorname{deg}(p) v_{p}(f) v_{p}(\operatorname{det} \varphi)} N_{k(p) / k}\left(\frac{f^{v_{p}(\operatorname{det} \varphi)}}{[\operatorname{det} \varphi]^{v_{p}(f)}}(p)\right) \in k^{*} .
$$

Proof. If $\varphi=\left(\begin{array}{cc}\operatorname{det} \varphi & 0 \\ 0 & 1\end{array}\right) \cdot \widetilde{\varphi}$ is the decomposition referred to above, it follows from the general properties of a commutator that

$$
\left\{\sigma_{f}, \varphi\right\}_{A_{p}^{2}}^{K_{2}^{2}}=\left\{\sigma_{f},\left(\begin{array}{cc}
\operatorname{det} \varphi & 0 \\
0 & 1
\end{array}\right)\right\}_{A_{p}^{2}}^{K_{2}^{2}} \cdot\left\{\sigma_{f}, \widetilde{\varphi}\right\}_{A_{p}^{2}}^{K_{p}^{2}}
$$


Accordingly, one has that

$$
\begin{aligned}
& \left\{\sigma_{f},\left(\begin{array}{cc}
\operatorname{det} \varphi & 0 \\
0 & 1
\end{array}\right)\right\}_{A_{p}^{2}}^{K_{p}^{2}}=\left\{\left(\begin{array}{cc}
f & 0 \\
0 & f
\end{array}\right),\left(\begin{array}{cc}
\operatorname{det} \varphi & 0 \\
0 & 1
\end{array}\right)\right\}_{A_{p}^{2}}^{K_{p}^{2}} \\
& =\left\{\left(\begin{array}{ll}
f & 0 \\
0 & 1
\end{array}\right),\left(\begin{array}{cc}
\operatorname{det} \varphi & 0 \\
0 & 1
\end{array}\right)\right\}_{A_{p}^{2}}^{K_{p}^{2}} \cdot\left\{\left(\begin{array}{cc}
1 & 0 \\
0 & f
\end{array}\right),\left(\begin{array}{cc}
\operatorname{det} \varphi & 0 \\
0 & 1
\end{array}\right)\right\}_{A_{p}^{2}}^{K_{2}^{2}} \\
& =\{f, \operatorname{det} \varphi\}_{A_{p}}^{K_{p}} \cdot(-1)^{i\left(f, A_{p}\right) \cdot i\left(\operatorname{det} \varphi, A_{p}\right)} \\
& =(-1)^{\operatorname{deg}(p) v_{p}(f) v_{p}(\operatorname{det} \varphi)} N_{k(p) / k}\left(\frac{f^{v_{p}(\operatorname{det} \varphi)}}{[\operatorname{det} \varphi]^{v_{p}(f)}}(p)\right) .
\end{aligned}
$$

Moreover, $\left\{\sigma_{f}, \widetilde{\varphi}\right\}_{A_{p}^{2}}^{K_{2}^{2}}=\left\{\sigma_{f}, J_{\widetilde{\varphi}}\right\}_{A_{p}^{2}}^{K_{p}^{2}}$, where $J_{\widetilde{\varphi}}$ is again the Jordan matrix associated with $\widetilde{\varphi}$, and

$$
J_{\widetilde{\varphi}} \in\left\{J^{1}:=\left(\begin{array}{cc} 
\pm 1 & 0 \\
1 & \pm 1
\end{array}\right), J^{2}:=\left(\begin{array}{cc}
0 & -1 \\
1 & \operatorname{tr} \widetilde{\varphi}
\end{array}\right), J^{3}:=\left(\begin{array}{cc}
g & 0 \\
0 & g^{-1}
\end{array}\right)\right\} .
$$

Our aim now is to see that $\left\{\sigma_{f}, J^{i}\right\}_{A_{p}^{2}}^{K_{2}^{2}}=1$ for $i \in\{1,2,3\}$.

Indeed, this assertion is a direct consequence of Lemma 4.5 for $J^{1}$ and $J^{2}$ with $\operatorname{tr} \widetilde{\varphi} \in A_{p}$.

Furthermore,

$$
\begin{aligned}
\left\{\sigma_{f}, J^{3}\right\}_{A_{p}^{2}}^{K_{p}^{2}} & =\left\{\left(\begin{array}{ll}
f & 0 \\
0 & 1
\end{array}\right),\left(\begin{array}{ll}
g & 0 \\
0 & 1
\end{array}\right)\right\}_{A_{p}^{2}}^{K_{2}^{2}} \cdot\left\{\left(\begin{array}{ll}
1 & 0 \\
0 & f
\end{array}\right),\left(\begin{array}{ll}
g & 0 \\
0 & 1
\end{array}\right)\right\}_{A_{p}^{2}}^{K_{p}^{2}} \\
& \cdot\left\{\left(\begin{array}{ll}
f & 0 \\
0 & 1
\end{array}\right),\left(\begin{array}{cc}
1 & 0 \\
0 & g^{-1}
\end{array}\right)\right\}_{A_{p}^{2}}^{K_{p}^{2}} \cdot\left\{\left(\begin{array}{cc}
1 & 0 \\
0 & f
\end{array}\right),\left(\begin{array}{cc}
1 & 0 \\
0 & g^{-1}
\end{array}\right)\right\}_{A_{p}^{2}}^{K_{p}^{2}} \\
& =\{f, g\}_{A_{p}}^{K_{p}} \cdot\left\{f, g^{-1}\right\}_{A_{p}}^{K_{p}} \cdot(-1)^{i\left(f, A_{p}\right) \cdot i\left(g, A_{p}\right)} \cdot(-1)^{i\left(f, A_{p}\right) \cdot i\left(g^{-1}, A_{p}\right)} \\
& =1 .
\end{aligned}
$$

Finally, since the general expression of $J^{2}$ is $\left(\begin{array}{cc}0 & -1 \\ 1 & h \cdot g^{-1}\end{array}\right)$, with $h, g \in A_{p}$ and $g \neq 0$, then

$$
\begin{aligned}
J^{2} & =\left(\begin{array}{cc}
g & 0 \\
0 & g^{-1}
\end{array}\right) \cdot\left(\begin{array}{cc}
0 & -g^{-1} \\
g & h
\end{array}\right) \\
& =\left(\begin{array}{cc}
g & 0 \\
0 & g^{-1}
\end{array}\right) \cdot\left[\left(\begin{array}{ll}
1 & 0 \\
0 & g
\end{array}\right) \cdot\left(\begin{array}{cc}
0 & -1 \\
1 & h
\end{array}\right) \cdot\left(\begin{array}{cc}
1 & 0 \\
0 & g^{-1}
\end{array}\right)\right]
\end{aligned}
$$

and, therefore,

$$
\left\{\sigma_{f}, J^{2}\right\}_{A_{p}^{2}}^{K_{p}^{2}}=\left\{\sigma_{f},\left(\begin{array}{cc}
g & 0 \\
0 & g^{-1}
\end{array}\right)\right\}_{A_{p}^{2}}^{K_{p}^{2}} \cdot\left\{\sigma_{f},\left(\begin{array}{cc}
0 & -1 \\
1 & h
\end{array}\right)\right\}_{A_{p}^{2}}^{K_{2}^{2}}=1 .
$$

Hence, $\left\{\sigma_{f}, \widetilde{\varphi}\right\}_{A_{p}^{2}}^{K_{2}^{2}}=1$ for every $\widetilde{\varphi} \in \operatorname{Gl}\left(2, \Sigma_{X}\right)$ such that $\operatorname{det} \widetilde{\varphi}=1$, whence the proposition is deduced.

Proposition 4.7. If $X$ is a complete, smooth and connected curve, for all $f \in \Sigma_{X}^{*}$ and $\varphi \in \operatorname{Gl}\left(2, \Sigma_{X}\right)$, one has that

$$
\prod_{p \in X}\left\{\sigma_{f}, \varphi\right\}_{A_{p}^{2}}^{K_{p}^{2}}=1
$$


Proof. The claim is a direct consequence of Theorem 4.2 and Lemma 4.4. because

$$
1=\prod_{p \in X}(-1)^{2 \operatorname{deg}(p) v_{p}(f) v_{p}(\operatorname{det} \varphi)}\left\{\sigma_{f}, \varphi\right\}_{A_{p}^{2}}^{K_{p}^{2}}=\prod_{p \in X}\left\{\sigma_{f}, \varphi\right\}_{A_{p}^{2}}^{K_{p}^{2}}
$$

Remark 4.8. If $(,)_{p}: \Sigma_{X}^{*} \times \Sigma_{X}^{*} \rightarrow k^{*}$ is the tame symbol, there exists a commutative diagram

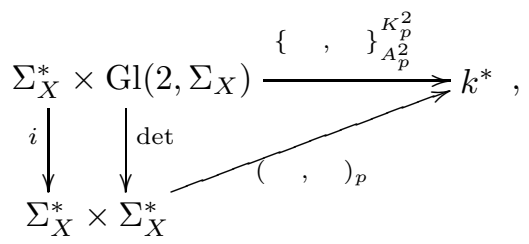

$i$ being the morphism $i\left(\sigma_{f}\right)=f$. A similar expression has recently been obtained by A. Beilinson, S. Bloch and H. Esnault ([5], 3.9.2) using super-extensions of $\Sigma_{X}^{*}$.

In particular, the restricted commutator map:

$$
\{, \quad\}_{A_{p}^{2}}^{K_{p}^{2}}: \Sigma_{X}^{*} \times \mathrm{Sl}\left(2, \Sigma_{X}\right) \rightarrow k^{*}
$$

is trivial, $\operatorname{Sl}\left(2, \Sigma_{X}\right)$ being the special linear group of $\Sigma_{X}$.

Therefore, the reciprocity law of Proposition 4.7 follows again from the Weil reciprocity law. A remaining problem is to determine whether the reciprocity law of $\operatorname{Gl}\left(2, \Sigma_{X}\right)$ (Theorem 4.2) can always be deduced from the Weil reciprocity law.

Remark 4.9 (Contou-Carrère Symbol on $\mathrm{Gl}\left(2, \Sigma_{X} \otimes_{k} B\right)$ ). Keeping the assumptions on $X$, if $B$ is a local Artinian $k$-algebra, the group $\operatorname{Gl}\left(2, \Sigma_{X} \otimes_{k} B\right)$ consists of the matrices:

$$
\tau \in \operatorname{Mat}_{2 \times 2}\left(\Sigma_{X} \otimes_{k} B\right),
$$

such that det $\tau$ is invertible in $\Sigma_{X} \otimes_{k} B$, and $\left(\Sigma_{X} \otimes_{k} B\right)^{*}$ is also a subgroup of $\operatorname{Gl}\left(2, \Sigma_{X} \otimes_{k} B\right)$ by means of the diagonal embedding $f \mapsto \sigma_{f}$. One has that $\operatorname{Gl}\left(2, \Sigma_{X} \otimes_{k} B\right) \subseteq \operatorname{Gl}\left(K_{p}^{2}\right)(\operatorname{Spec} B)$ and there exists a central extension of groups

$$
1 \rightarrow B^{*} \longrightarrow \widetilde{\mathrm{Gl}}\left(2, \Sigma_{X} \otimes_{k} B\right) \longrightarrow \mathrm{Gl}\left(2, \Sigma_{X} \otimes_{k} B\right) \rightarrow 1,
$$

whose commutator is denoted by $\{\varphi, \phi\}_{A_{p, B}^{2}}^{K_{p, B}^{2}}$, for all $\varphi, \phi \in \operatorname{Gl}\left(2, \Sigma_{X} \otimes_{k} B\right)$, such that $\varphi \cdot \phi=\phi \cdot \varphi$.

We have a map $\{\quad, \quad\}_{A_{p, B}^{2}}^{K_{p}^{2}}:\left(\Sigma_{X} \otimes_{k} B\right)^{*} \times \operatorname{Gl}\left(2, \Sigma_{X} \otimes_{k} B\right) \rightarrow B^{*}$ and, similar to the above and using the statements of [3], a computation shows that $\left\{\sigma_{f}, \varphi\right\}_{A_{p, B}^{2}}^{K_{2}^{2}}=$ $\langle f \text {, det } \varphi\rangle_{p}$, where $\langle, \quad\rangle_{p}$ is the Contou-Carrère symbol $([7]), f$ and $\operatorname{det} \varphi$ being Laurent series with coefficients in $B$ via an immersion $\left(\Sigma_{X} \otimes_{k} B\right)^{*} \hookrightarrow B((t))^{*}$.

Moreover, when $X$ is complete, using the method of this work a hypothetical generalization of the abstract reciprocity law (Theorem 3.5) to Spec $B$-valued points will allow us to recover the reciprocity law for the Contou-Carrère symbol of an algebraic curve over a perfect field (see [10]) without assuming that $B$ is a finite $k$-algebra. 


\section{REFERENCES}

[1] Álvarez, A., Drinfeld Moduli Schemes and Infinite Grassmannians, J. Algebra 225, (2000) 822-835. MR 1741564(2001e:11059a)

[2] Álvarez Vázquez, A.; Muñoz Porras, J. M.; Plaza Martín, F. J., The Algebraic Formalism of Soliton Equations over Arbitrary Base Fields, Aportaciones Matemáticas: Taller de Variedades Abelianas y Funciones Theta; Sociedad Matemática Mexicana 13, (1998) 3-40. MR 1781698 (2002d:14051)

[3] Anderson, G. W.; Pablos Romo, F., Simple Proofs of Classical Explicit Reciprocity Laws on Curves using Determinant Groupoids over an Artinian Local Ring, Comm. Algebra 32(1), (2004) 79-102. MR2036223 (2005d:11099)

[4] Arbarello, E.; de Concini, C.; Kac, V.G., The Infinite Wedge Representation and the Reciprocity Law for Algebraic Curves, Proc. of Symposia in Pure Mathematics, Volume 49, Part I, A.M.S., (1989) 171-190. MR.1013132(90i:22034)

[5] Beilinson, A.; Bloch S.; Esnault H., $\epsilon$-factors for Gauss-Manin determinants, Moscow Math. J. 2(3), (2002) 477-532. MR1988970 (2004m:14011)

[6] Beilinson, A.; Drinfeld, V., Quantization of Hitchin's Integrable System and Hecke Eigensheaves, manuscript available at http://www.math.uchicago.edu/ arinkin/langlands/.

[7] Contou-Carrère, C., Jacobienne Locale, Groupe de Bivecteurs de Witt Universel, et Symbole Modéré, C.R. Acad. Sci. Paris, t. 318, Série I (1994) 743-746. MR1272340 (95c:14059)

[8] Kapranov, M., Semiinfinite symmetric powers, e-print: math.QA/0107089.

[9] Knudsen, F.; Mumford, D., The projectivity of the moduli space of stable curves I: Preliminaries on det and Div., Math. Scand. 39, (1976) 19-55. MR0437541 (55:10465)

[10] Pablos Romo, F., A Generalization of the Contou-Carrère Symbol, Israel J. Math. 141, (2004) 39-60. MR2063024 (2005g:11115)

[11] Pablos Romo, F., On the Tame Symbol of an Algebraic Curve, Comm. Algebra 30(9), (2002) 4349-4368. MR1936475 (2003k:14042)

[12] Pressley, A.; Segal, G., Loop Groups, Oxford Univ. Press, Oxford (1986). MR900587 (88i:22049)

[13] Segal, G.; Wilson, G., Loop groups and equations of KdV type, I.H.E.S., Publications Mathématiques 61, (1985) 5-64. MR783348 (87b:58039)

[14] Tate, J., Residues of Differentials on Curves, Ann. Scient. École. Norm. Sup., 4a série 1, (1968) 149-159. MR0227171 (37:2756)

[15] Weil, A., Généralisation des fonctions abéliennes, J. Math. Pures et Appl. 17, (1938) 47-87.

[16] Witten, E., Quantum Field Theory, Grassmannians and Algebraic Curves, Comm. Math. Phys. 113, (1988) 529-600. MR923632 (88m:81127)

Departamento de Matemáticas, Universidad de Salamanca, Plaza de la Merced 1-4, Salamanca 37008, Spain

E-mail address: jmp@usal.es

Departamento de Matemáticas, Universidad de Salamanca, Plaza de la Merced 1-4, Salamanca 37008, Spain

E-mail address: fpablos@usal.es 